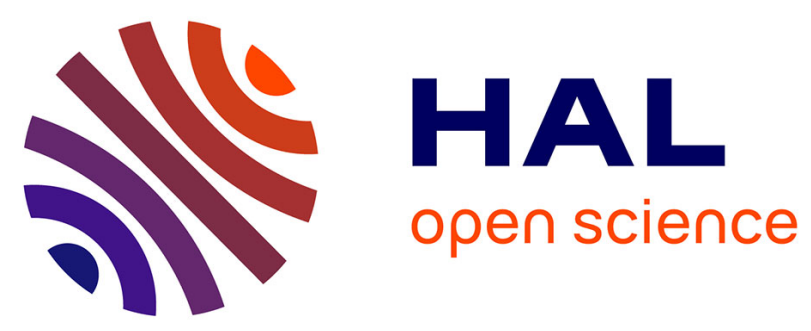

\title{
Humans are able to self-paced constant running accelerations until exhaustion
}

Véronique Billat, Nicolas J-B Brunel, Thomas Carbillet, Stéphane Labbé, Adeline Samson

\section{- To cite this version:}

Véronique Billat, Nicolas J-B Brunel, Thomas Carbillet, Stéphane Labbé, Adeline Samson. Humans are able to self-paced constant running accelerations until exhaustion. Physica A: Statistical Mechanics and its Applications, 2018, 506, pp.290-304. 10.1016/j.physa.2018.04.058 . hal-01965678

\section{HAL Id: hal-01965678 \\ https://hal.science/hal-01965678}

Submitted on 26 Dec 2018

HAL is a multi-disciplinary open access archive for the deposit and dissemination of scientific research documents, whether they are published or not. The documents may come from teaching and research institutions in France or abroad, or from public or private research centers.
L'archive ouverte pluridisciplinaire $\mathbf{H A L}$, est destinée au dépôt et à la diffusion de documents scientifiques de niveau recherche, publiés ou non, émanant des établissements d'enseignement et de recherche français ou étrangers, des laboratoires publics ou privés. 


\title{
Humans are able to self-paced constant running accelerations until exhaustion
}

\author{
Véronique Billat ${ }^{1}$, Nicolas J-B. Brunel ${ }^{2}$, Thomas Carbillet ${ }^{* 34}$, Stéphane Labbé ${ }^{3}$ \\ and Adeline Samson ${ }^{3}$ \\ ${ }^{1}$ Unité de Biologie Intégrative des Adaptations à l'Exercice, Université \\ Paris-Saclay, Evry, France \\ ${ }^{2}$ LaMME, ENSIIE, CNRS, UEVE, Université Paris-Saclay, Evry, France \\ ${ }^{3}$ Université Grenoble Alpes, Laboratoire Jean Kuntzmann, Grenoble, France \\ ${ }^{4}$ Billatraining SAS
}

April 13, 2018

\begin{abstract}
Although it has been experimentally reported that speed variations is the optimal way of optimizing his pace for achieving a given distance in a minimal time, we still do not know what the optimal speed variations (i.e accelerations) are. At first, we have to check the hypothesis that human is able to accurately self-pacing its acceleration and this even in a state of fatigue during exhaustive self-pacing ramp runs. For that purpose, 3 males and 2 females middle-aged, recreational runners ran, in random order, three exhaustive acceleration trials. We instructed the five runners to perform three self-paced acceleration trials based on three acceleration intensity levels: "soft", "medium" and "hard". We chose a descriptive modeling approach to analyse the behaviour of the runners. Once we knew that the runners were able to perceive three acceleration intensity levels, we proposed a meanreverting process (Ornstein-Uhlenbeck) to describe those accelerations: $d a_{t}=-\theta\left(a_{t}-a\right) d t+$ $\sigma d W_{t}$ where $a$ is the mean acceleration, $a_{t}$ is the measured acceleration at each time interval
\end{abstract}

*Corresponding author: thomas.carbillet@gmail.com

Thomas Carbillet, 19 chemin de Lagay, 38320 Eybens, France 
$t, \theta$ the ability of the runner to correct the variations around a mean acceleration and $\sigma$ the human induced variations. The goodness-of-fit of the Ornstein-Uhlenbeck process highlights the fact that humans are able to maintain a constant acceleration and are able to precisely regulate their acceleration (regardless of its intensity) in a run leading to exhaustion in the range from 1 minutes 36 seconds to 20 minutes.

\section{Keywords}

Acceleration; Exercise; Running; Self-paced; Performance; Ornstein-Uhlenbeck

\section{Introduction}

Endurance running is considered to have played a major role in human evolution. Humans have developed the ability to fine-tune their running speed in order to run for several days and still catch their fastest prey [1]. Indeed, it has been reported that speed variation is the ideal way of optimizing its pace for achieving a given distance in a minimal time [2, $3,4,4,6,6$, 8. 9. Hence, the rate at which the velocity of a body changes with time requires positive and negative accelerations and then strength variations according to Newton's second law of motion. However, there is a direct relationship between force impulse and running acceleration [10] and the minimum-jerk model [11] predicts that to save energy and optimize performance, running must be as smooth as possible and variations in acceleration must be close to $0 \mathrm{m.s}^{-3}$. Furthermore, there is a clearly non-random speed fluctuation during self-paced exercise [12, 13, 14, 15]. It has been suggested that speed variability operates through a feed forward mechanism [16] and we know that most of the races are stochastic when the velocity is not imposed [17]. Despite the large published body of work on pacing strategy and speed control, this is the first study to have examined acceleration control during running. We know that the fastest runners on middle and long-distance run with light speed variations [18, 19, 20]. Now that sensors make the recording of acceleration data easy, we would like to check if humans are able to integrate instructions in acceleration. The purpose is to take part in a runner's training and race in future studies. Given the fact that we are looking for a stochastic process to describe the ability for humans to maintain a constant acceleration and the hypothesis that self-paced accelerations lightly fluctuate around a constant value, we thought about the Ornstein-Uhlenbeck process [21]. Such process is described as a stochastic process with a mean-reverting property. The use of Ornstein-Uhlenbeck process 
is present in fields such as mathematical finance 22] (volatilities of asset prices and dynamics of interest rates) and biological processes [23]. Before going deeper in the effective mechanism of speed variation control during exhaustive exercise, we have to verify human's ability to maintain constant acceleration in a conscious way until exhaustion. The present study tests the hypothesis whereby humans are able to maintain a constant acceleration in a self-paced trial with no external cues regardless of the speed and the magnitude of acceleration and fatigue. For that purpose, we study more specifically the following two points:

(1) The ability for humans to maintain three different intensity levels of constant acceleration when so instructed. Those three intensities are described as "soft", "medium" and "hard" which are the instructions we gave to the runners for respectively a slow, a medium and a hard constant acceleration.

(2) The characterization of the acceleration data by introducing a new interpretation using a mean-reversing process (Ornstein-Uhlenbeck).

The paper is organized as follows. Section 2 describes the experimental protocol and presents the mathematical model. Section 3 compares the three acceleration intensities for each runner to see if we notice a genuine difference and gives an estimation of the parameters of the OrnsteinUhlenbeck model for characterizing human accelerations. Finally, section 4 and 5 conclude the paper.

\section{Methods}

\section{$2.1 \quad$ Subjects}

The study population is composed of three males and two females recreational runners (aged $38 \pm 3$ yrs., total running distance per week: $36.1 \pm 4.3 \mathrm{~km}$; body weight: $66.9 \pm 12.4 \mathrm{~kg}$ and height $171.1 \pm 6.7 \mathrm{~cm})$. All subjects were first informed of the risks and constraints associated with the protocol and gave their written, informed consent to participation. The present study conformed to the precepts of the Declaration of Helsinki and all procedures were approved by the local investigational review board (Saint Louis Hospital, Paris, France). On a physiological aspect, we cannot compare openly males and females. Given this fact plus the sample size of the study, we choose to focus on a descriptive modeling approach to analyse the runner behaviours. No statistical tests will be applied. 


\subsection{Experimental Design}

Subjects ran alone and performed three types of track sessions until exhaustion, in random order and with a one-hour interval between sessions: the first, second and third track tests were selfpaced acceleration trials at respectively slow, medium and high accelerations. We characterize those accelerations as "soft", "medium" and "hard". Velocity and acceleration were measured with a GPS-enabled accelerometer of 10 and $100 \mathrm{~Hz}$ the Minimax from Catapult Sports (Pty Ltd, Victoria, Australia). The GPS and accelerometer signals were sampled at 5 and $50 \mathrm{~Hz}$, respectively, and averaged per second. The difference between the real distance (track) and the recorded distance (GPS) was less than $1 \%$ and $0.92 \%$ over $800 \mathrm{~m}$ and $1500 \mathrm{~m}$, respectively. This result agrees with previous GPS studies for maximal efforts run by humans or horses [24, 25].

\subsection{Acceleration trials}

In the self-paced acceleration trial protocol, the runners performed three freely paced acceleration sets in which they were asked to maintain constant acceleration by progressively increasing their speed until exhaustion. The trials were run at three constant acceleration values, based on ratings of perceived acceleration ("soft", "medium" and "hard"), in random order. In all of the the selfpaced acceleration trials, the runners started at an initial velocity between 2.2 and 3.05 m.s and then increased their velocity at three different, constant accelerations ("soft", "medium" and "hard" intensities). There was a one-hour interval between acceleration trials. In the self-paced acceleration trials set, the runners were not provided with any external information other than the distance covered. All tests were performed between $3 \mathrm{pm}$ and $6 \mathrm{pm}$ on wind-free, spring days $\left(<2 \mathrm{~m} . \mathrm{s}^{-1}\right.$ according to an anemometer, the Windwatch from Alba, Silva, Sweden) with a temperature of $20^{\circ} \mathrm{C}$, as in a previous study of the energetics of middle-distance running [26].

\subsection{Mathematical Model}

We know that most of the races are stochastic when the velocity is not imposed [17. Our hypothesis analyzing the measured data was that humans can maintain a constant acceleration with little variations around a mean value. Therefore, we are characterizing the human acceleration on self-paced exercises with three different instruction intensities ("soft", "medium" and "hard") with a Ornstein-Uhlenbeck process. From the model to the simulation, this subsection explains the method used for characterizing the runners self-paced acceleration when so instructed. 


\subsubsection{The Ornstein-Uhlenbeck process}

To assess the subjects' ability to maintain self-paced, constant acceleration, we check that the equation for constant-acceleration motion is satisfied. If the acceleration of the runner $(a)$ is constant then the runner's speed at time $\mathrm{t}$, denoted $v(t)$, is a linear function of time $v(t)=$ $v(0)+a t$, where $v(0)=v_{0}$ is the initial speed. Here, $v_{0}$ is within the range of 2.2 to 3.05 $m . s^{-1}$. As a consequence, the distance $d(t)$ covered at time $t$ has a closed form obtained directly by integration as $d(t)=0.5 a t^{2}+v_{0} . t$ if we consider that $d(0)=0$. Hence, the theoretical relationship between distance and time is a polynomial of second order. As mentioned in the introduction, in order to save energy and optimize performance, running must be as smooth as possible (variation in acceleration must be close to $0 \mathrm{~m} . \mathrm{s}^{-3}$ ) during self-paced exercise. In this study, we want to see if the following analogy is possible: self-paced acceleration is as smooth as possible during self-paced exercise. Let us denote $a(t)$ the acceleration at time $t$. The objective of the paper is to propose a reasonable stochastic model for $a(t)$. Assuming that each runner has an internal variability and an autocorrect ability, we define $a(t)$ as the solution of:

$$
d a(t)=-\theta[a(t)-a] d t+\sigma d W(t)
$$

The above equation is the definition of a mean-reverting process also called Ornstein Uhlenbeck process. $a(t)\left(m . s^{-2}\right)$ is the acceleration of the runner at time $t(s) . a\left(m . s^{-2}\right)$ is the mean acceleration. We distinguish two human factors, $\theta$ depicting the autocorrect ability of the runner (that is also the speed reversion parameter) and $\sigma$ the human variability (that is also the short term standard deviation). The stochastic nature of the model is brought by the Brownian motion $W(t)$.

A solution in terms of integral of a mean-reverting process can be written as the following equation [27]:

$$
a(t)=a(0) e^{-\theta t}+a\left(1-e^{-\theta t}\right)+\sigma e^{-\theta t} \int_{0}^{t} e^{\theta s} \mathrm{~d} W(s)
$$

The three parameters $a, \theta$ and $\sigma$ are unknown. The objective is to estimate them from the data and to validate a posteriori the fit obtained with model (1). From equation (2), we would like to highlight the fact that unlike the beginning of the current subsection $(2.4 .1), a(t)$ is not consistent anymore. In addition $a(t)$ and $v(t)$ are not linear in time. The next subsection focuses on the estimation of the parameters $a, \theta$ and $\sigma$ from the data. 


\subsubsection{Parameter estimation}

Parameter estimation from discrete observation $a(i \Delta)$ for $i=0, . ., n$ can be performed by optimizing a least square criteria. Indeed, we have

$$
a(t)=e^{-\theta \Delta t} a\left(t-\Delta_{t}\right)+\left(1-e^{-\theta \Delta_{t}}\right) a+\sigma \sqrt{\frac{1-e^{-2 \theta \Delta_{t}}}{2 \theta}}\left(W(t)-W\left(t-\Delta_{t}\right)\right)
$$

Let us introduce a new parametrization: $\alpha=\left(1-e^{-\theta \Delta_{t}}\right) a, \beta=e^{-\theta \Delta_{t}}$ and $\gamma=\sigma \sqrt{\frac{1-e^{-2 \theta \Delta_{t}}}{2 \theta}}$ The least square criteria is then defined as $(\hat{\alpha}, \hat{\beta})=\underset{(\alpha, \beta)}{\operatorname{argmin}} \sum_{i=1}^{n}(a(i \Delta)-\beta a((i-1) \Delta)-\alpha)^{2}$ and $\hat{\gamma}^{2}=\frac{1}{n} \sum_{i=1}^{n}(a(i \Delta)-\hat{\beta} a((i-1) \Delta)-\hat{\alpha})^{2}$. The estimators correspond to the maximum likelihood estimators. They are consistent, unbiased and asymptotically normal. The solution of $\hat{\alpha}$ and $\hat{\beta}$ are explicit. The Fisher information matrix can be computed explicitly, that corresponds to the asymptotic variance denoted $\hat{\sum}$.

To obtain the estimation of the original parameters, we use the following transformation: $\phi(\alpha, \beta, \gamma)=$

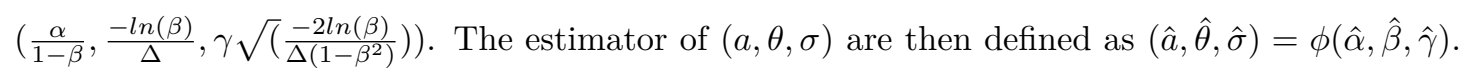
By the delta method, we can prove that their are asymptotically normal: $(\hat{a}, \hat{\theta}, \hat{\sigma}) \stackrel{\mathcal{L}}{\rightarrow} \mathcal{N}\left(\left(a^{*}, \theta^{*}, \sigma^{*}\right), \mathcal{J}_{\phi} \hat{\sum} \mathcal{J}_{\phi}^{\prime}\right)$ where $\left(a^{*}, \theta^{*}, \sigma^{*}\right)$ are the true (unknown) parameters and $\mathcal{J}_{\phi}$ is the Jacobian of $\phi$. This allows to compute confidence interval for $(a, \theta, \sigma)$.

\section{Results}

\subsection{Comparison of the three different acceleration exercises}

In order to know if humans are able to perceive different intensity levels of acceleration, we instructed the five runners to perform three self-paced constant acceleration trials (see subsection 2.3). The result of the three trials for the first male runner is shown in Figure 1 .

On each plot, the velocity corresponding to "hard" intensity has the highest slope, the "medium" intensity has the medium slope and the "soft" intensity acceleration trial has the smallest one. Results of the four other runners can be found in the appendix A, Figures 4, 5, 6, 7, Table 1 presents the distance and time at exhaustion for each runner and each trial.

The three different intensity levels are distinct for each runner. The times to exhaustion are in the range $96 s$ (1 minute and 36 seconds) to $1204 s$ (approximately 20 minutes). The average time to exhaustion for "soft" intensities is 677.6 seconds (approximately 11 minutes and 6 seconds), for "medium" intensities is 368.6 seconds (6 minutes and 26 seconds) and for "hard" intensities is 169.8 seconds (2 minutes and 49 seconds). 


\begin{tabular}{|c|c|c|c|}
\hline \multicolumn{2}{|c|}{ Trial } & Final Distance $(\mathrm{m})$ & Final Time $(\mathrm{s})$ \\
\hline \multirow{4}{*}{ Soft } & Male 1 & 1120 & 409 \\
\cline { 2 - 4 } & Male 2 & 4403 & 1204 \\
\cline { 2 - 4 } & Male 3 & 1718 & 602 \\
\cline { 2 - 4 } & Female 1 & 1931 & 603 \\
\cline { 2 - 4 } & Female 2 & 1605 & 570 \\
\hline \hline \multirow{2}{*}{ Average "Soft" } & 2155.4 & 677.6 \\
\hline \hline \multirow{4}{*}{ Medium } & Male 1 & 888 & 296 \\
\cline { 2 - 4 } & Male 2 & 2359 & 595 \\
\cline { 2 - 4 } & Male 3 & 923 & 282 \\
\cline { 2 - 4 } & Female 1 & 1139 & 333 \\
\cline { 2 - 4 } & Female 2 & 1029 & 337 \\
\hline \hline \multirow{7}{*}{ Average "Medium" } & 1267.6 & 368.6 \\
\hline \hline \multirow{2}{*}{ Hard } & Male 1 & 491 & 143 \\
\cline { 2 - 4 } & Male 2 & 762 & 166 \\
\cline { 2 - 4 } & Male 3 & 436 & 96 \\
\cline { 2 - 4 } & Female 1 & 832 & 225 \\
\cline { 2 - 4 } & Female 2 & 756 & 169.8 \\
\hline \hline \multirow{7}{*}{ Average "Hard" } & 655.4 & \\
\hline
\end{tabular}

Table 1 - Distance and Time at exhaustion for each runner and for each acceleration trial intensity: "soft", "medium" and "hard"

\begin{tabular}{|l|c|c|c||c||c|c||c|}
\hline & Male 1 & Male 2 & Male 3 & Average Males & Female 1 & Female 2 & Average Females \\
\hline$\frac{\text { soft }}{\text { medium }}$ & 1.26 & 1.87 & 1.86 & 1.66 & 1.70 & 1.56 & 1.63 \\
\hline$\frac{\text { medium }}{\text { hard }}$ & 1.81 & 3.09 & 2.21 & 2.37 & 1.37 & 1.36 & 1.37 \\
\hline
\end{tabular}

Table 2 - Ratios of distances to exhaustion for "soft" vs. "medium" and "medium " vs. "hard" acceleration intensities for each runner 

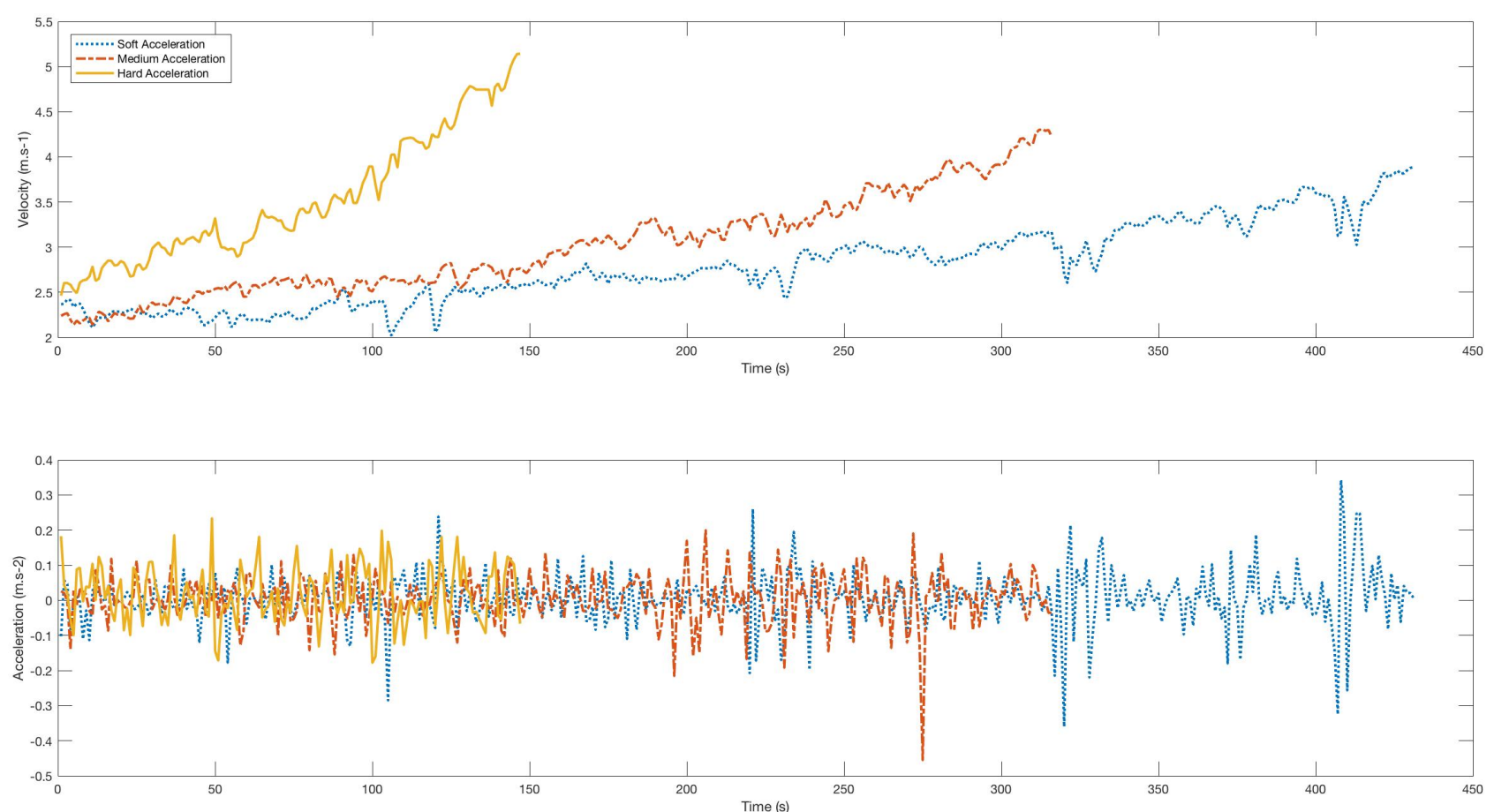

Figure 1 - Male Runner 1 - Top chart: Velocity (m/s) VS. Time (s); Bottom chart: Acceleration $\left(\mathrm{m} / \mathrm{s}^{2}\right)$ VS. Time (s) - Comparison of the three acceleration intensity instructions: "soft" (blue curve), "medium" (red curve) and "hard" (yellow curve)

Moreover the average ratios of the "Soft" to "Medium" instructions on "Distance" and "Time" are respectively 1.65 and 2.22 and the average ratios of the "Medium" to "Hard" instructions on "Distance" and "Time" are respectively 1.81 and 2.32. Tables 2 and 3 display those results. Note that we do not apply any statistical test to determine the significativity of the three regimes because of the low sample size ( 3 males and 2 females). This highlights the fact that humans are able to feel three different intensities of acceleration and divide distance and time to exhaustion from one intensity to another.

The fact that the three regimes are clearly distinct on the plot strengthens the idea that humans are able to maintain at least three different intensities of acceleration. 


\begin{tabular}{|l|c|c|c||c||c|c||c|}
\hline & Male 1 & Male 2 & Male 3 & Average Males & Female 1 & Female 2 & Average Females \\
\hline$\frac{\text { soft }}{\text { medium }}$ & 1.38 & 2.02 & 2.13 & 1.84 & 1.81 & 1.69 & 1.75 \\
\hline$\frac{\text { medium }}{\text { hard }}$ & 2.07 & 3.58 & 2.94 & 2.86 & 1.48 & 1.54 & 1.51 \\
\hline
\end{tabular}

Table 3 - Ratios of times to exhaustion for "soft" vs. "medium" and "medium" vs. "hard" acceleration intensities for each runner

\subsection{Characterizing the human faculty to maintain a constant acceler- ation}

As shown before, we can consider that humans are able to perceive different intensity levels of acceleration. The objective is how to characterize those accelerations. To do so, the OrnsteinUhlenbeck model (3) is applied.

The goal is to show that it is possible to characterize the human acceleration. We would like to give an estimation and interpretation of the parameters of the Ornstein-Uhlenbeck model $(\hat{a}, \hat{\theta}, \hat{\sigma})$ and their confidence interval. By applying the method described in subsection 2.4.2, we have estimated $\hat{a}, \hat{\theta}$ and $\hat{\sigma}$ and the confidence interval for $\hat{a}$. Results of the estimated parameters and confidence intervals for $\hat{a}$ are shown in Table 4

We observe the following two points from Table 4 ;

1. The mean autocorrect abilities $(\hat{\theta})$ of the runners are steady with a slight drop in the "Hard" trial. This means that the runners are less able to correct their mistakes during the "Hard" trials.

2. The human variability $(\hat{\sigma})$ is steady too with a slight rise in the "Hard" trial. This could be an answer to the drop of autocorrect ability. This means that the runner variability tends toward increasing during "Hard" trial.

The next step consists in checking if our model could explain the reality. By its very nature, an Ornstein-Uhlenbeck process is Gaussian. This property allows us to compute the quantiles exactly. Once such confidence boundaries are computed, we check the percentage of the measured acceleration data present inside.

Ratios of estimated acceleration for "soft" vs. "medium" and "medium" vs. "hard" acceleration intensities in Table 5 seem to confirm the hypothesis that humans are able to distinct at least 3 intensities of acceleration. 


\begin{tabular}{|c|c|c|c|c|c|}
\hline \multicolumn{2}{|c|}{ Trial } & $\hat{a}$ & $\hat{\theta}$ & $\hat{\sigma}$ & C.I \\
\hline \multirow[t]{5}{*}{ Soft } & Male 1 & 0.0036 & 1.9803 & 0.1188 & {$[0.0032 ; 0.0039]$} \\
\hline & Male 2 & 0.0026 & 1.3763 & 0.0827 & {$[0.0025 ; 0.0027]$} \\
\hline & Male 3 & 0.0046 & 1.4311 & 0.0868 & {$[0.0044 ; 0.0048]$} \\
\hline & Female 1 & 0.0038 & 1.6404 & 0.0600 & {$[0.0037 ; 0.0039]$} \\
\hline & Female 2 & 0.0033 & 1.4077 & 0.0713 & {$[0.0031 ; 0.0035]$} \\
\hline \multicolumn{2}{|c|}{ Average "Soft" } & 0.0036 & 1.5672 & 0.0839 & - \\
\hline \multirow[t]{5}{*}{ Medium } & Male 1 & 0.0049 & 1.5569 & 0.1008 & {$[0.0045 ; 0.0054]$} \\
\hline & Male 2 & 0.0040 & 1.2028 & 0.0818 & {$[0.0037 ; 0.0042]$} \\
\hline & Male 3 & 0.0103 & 1.9777 & 0.1372 & {$[0.0098 ; 0.0109]$} \\
\hline & Female 1 & 0.0066 & 1.6293 & 0.0565 & {$[0.0063 ; 0.0068]$} \\
\hline & Female 2 & 0.0047 & 1.7663 & 0.0669 & {$[0.0045 ; 0.0050]$} \\
\hline \multicolumn{2}{|c|}{ Average "Medium" } & 0.0061 & 1.6266 & 0.0886 & - \\
\hline \multirow[t]{5}{*}{ Hard } & Male 1 & 0.0172 & 1.0275 & 0.0982 & {$[0.0159 ; 0.0185]$} \\
\hline & Male 2 & 0.0160 & 1.4493 & 0.1054 & {$[0.0151 ; 0.0169]$} \\
\hline & Male 3 & 0.0253 & 0.7115 & 0.1400 & {$[0.0213 ; 0.0293]$} \\
\hline & Female 1 & 0.0088 & 1.2892 & 0.0450 & {$[0.0085 ; 0.0091]$} \\
\hline & Female 2 & 0.0065 & 1.3800 & 0.0907 & {$[0.0059 ; 0.0072]$} \\
\hline \multicolumn{2}{|c|}{ Average "Hard" } & 0.0147 & 1.1715 & 0.0958 & - \\
\hline
\end{tabular}

Table 4 - Mean acceleration ( $\hat{a})$, autocorrect ability $(\hat{\theta})$ and human variability $(\hat{\sigma})$ estimated for each runner and for each acceleration trial intensity: "soft", "medium" and "hard" and confidence interval (C.I) for $\hat{a}$

\begin{tabular}{|c|c|c|c|c|c||c|}
\hline & Male 1 & Male 2 & Male 3 & Female 1 & Female 2 & Average \\
\hline$\frac{\text { soft }}{\text { medium }}$ & 0.73 & 0.65 & 0.45 & 0.56 & 0.70 & 0.62 \\
\hline$\frac{\text { medium }}{\text { hard }}$ & 0.28 & 0.25 & 0.41 & 0.75 & 0.72 & 0.48 \\
\hline
\end{tabular}

Table 5 - Ratios of estimated acceleration for "soft" vs. "medium" and "medium" vs. "hard" acceleration intensities for each runner 
In order to evaluate the validity of the mean-reverting process applied to acceleration, the goodness-of-fit of the model has been tested in three steps:

1. Checking the ability of the Ornstein-Uhlenbeck model to distinguish three distinct mean accelerations.

2. Checking for non autocorrelation of residuals with the Durbin-Watson test.

3. Testing for stationarity with the Augmented Dickey-Fuller (ADF) Test.

\subsubsection{Checking the ability of the Ornstein-Uhlenbeck model to distinguish three distinct mean accelerations}

Let us now highlight the ability of our model to distinguish the three intensities of acceleration. In order to do so, we use the delta method to compute a confidence interval for each estimated acceleration $\hat{a}$. The Figure 2 displays the three different estimated mean accelerations for the male runner 1 , symbolized by three dots. The $95 \%$ confidence intervals for each estimated mean acceleration have been plotted as well symbolizing by error bars. It appears that the suggested Ornstein-Uhlenbeck model seems to distinguish three distinct mean accelerations: Figure 2 and Figures $12,13,14,15$ for the four other runners show that the confidence intervals do not overlap. However, because of the low sample size, this conclusion is purely descriptive. A statistical test will be applied on the future on a larger dataset. 


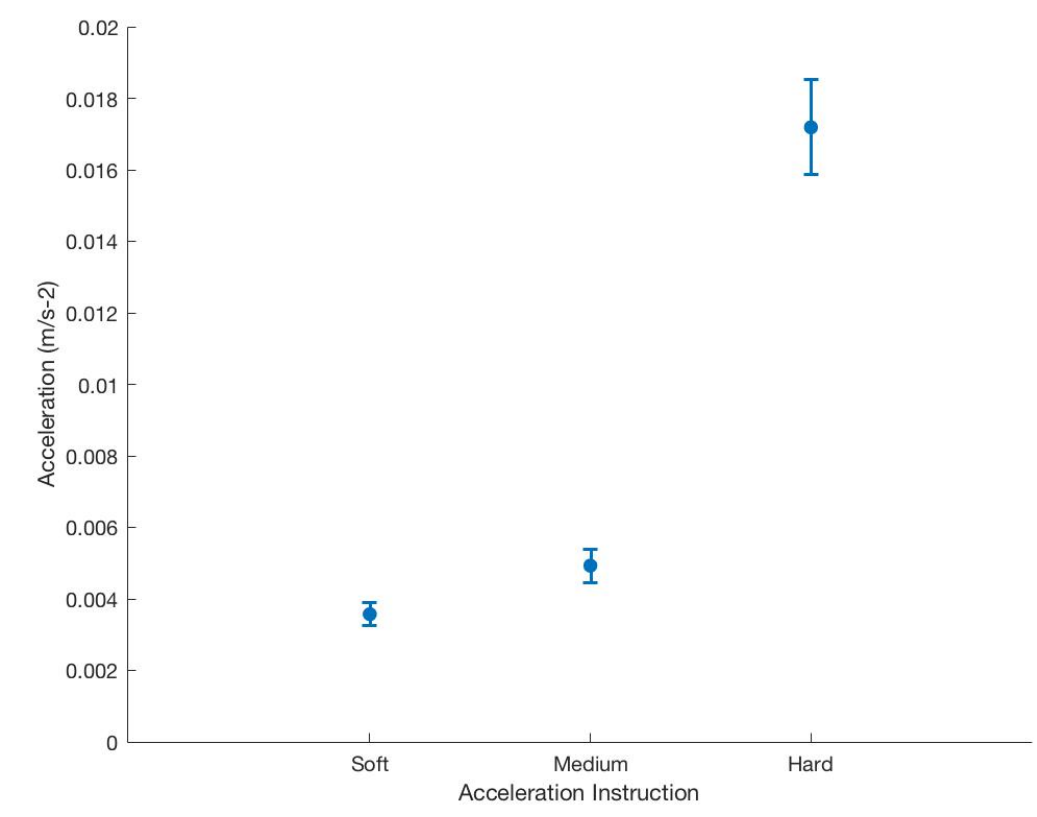

Figure 2-Male Runner 1 - Comparison of the three estimated mean accelerations

The Figure 3 shows the result of one simulation for the first male runner. We can distinguish 3 information per chart with two curves and one filled region on each chart. The black curve (dotted) represents the measured data (i.e. measured accelerations), the red one (solid) represents one random simulation of the Ornstein-Uhlenbeck model (equation (3) subsection 2.4) and the filled region stands for the $95 \%$ confidence interval.

We can quickly observe that the confidence interval is consistent with the reality of our data and that most of the recorded acceleration data seem to be inside the confidence interval. Results for the four other runners can be found in appendix B, Figures 8 to 11

To confirm that a significant amount of measured acceleration are inside the confidence boundaries, Table 6 shows that the values of percentages of measured data found in the $95 \%$ confidence boundaries are above $92 \%$. Thus the Ornstein-Uhlenbeck model described in subsection 2.4.2 seems to be consistent and could be used to describe the reality.

To go further, it would be interesting to perform self-paced acceleration following the same protocol with first, more runners in order to check if there is a trend in the ratios, second, splitting the runners into two categories (men and women) to check if there is a gender effect on those ratios. 

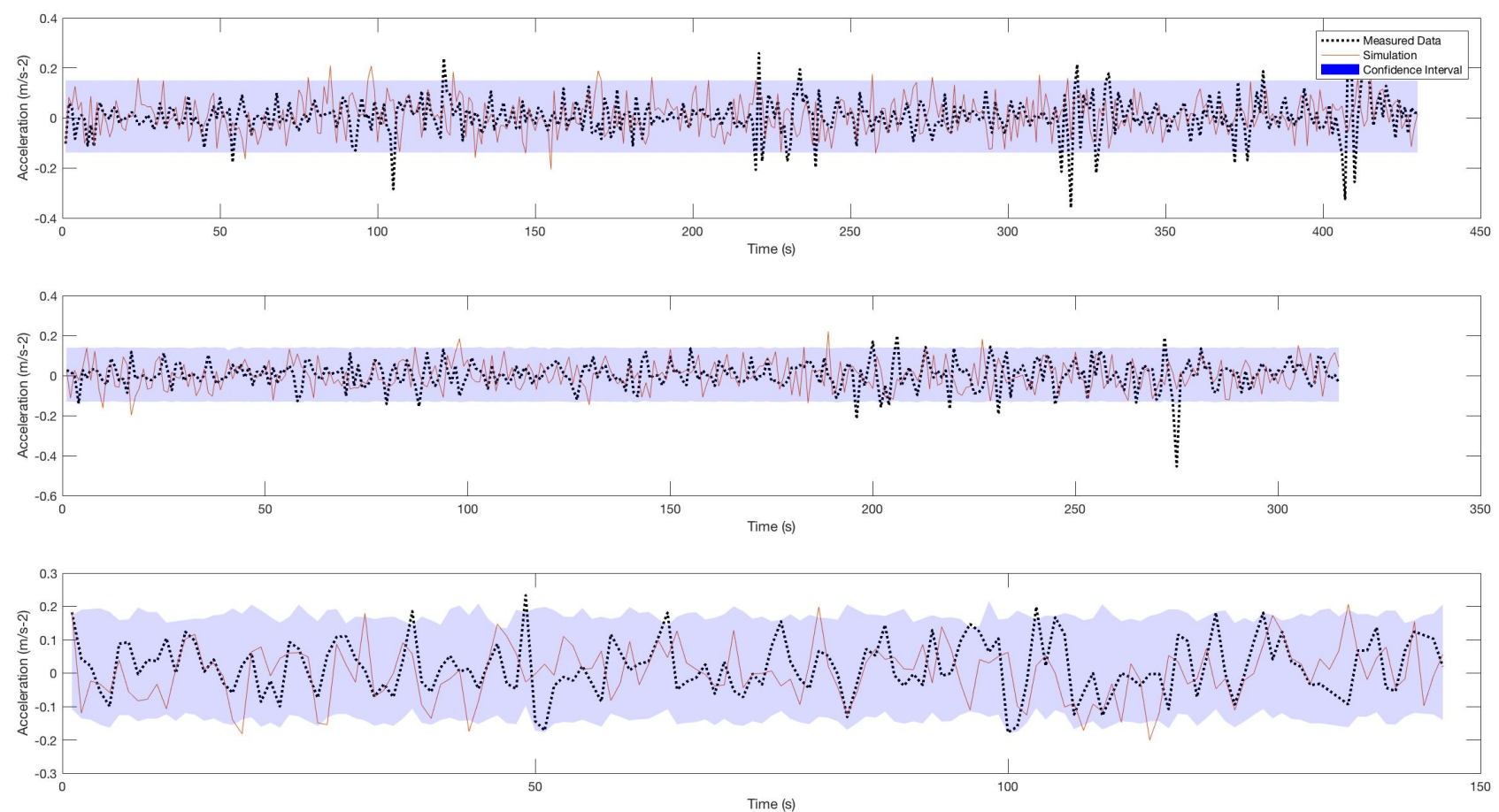

Figure 3 - Male Runner 1 - From top to bottom: Soft, Medium and Hard Acceleration Intensities Measured data VS. Ornstein-Uhlenbeck model

\begin{tabular}{|c|c|c|c|c|c|c|}
\hline \multicolumn{2}{|c|}{} & Male 1(\%) & Male 2(\%) & Male 3(\%) & Female 1(\%) & Female 2(\%) \\
\hline \multirow{3}{*}{ Acceleration } & Soft & 94.42 & 95.43 & 94.02 & 97.51 & 97.89 \\
\cline { 2 - 7 } & Medium & 95.24 & 97.14 & 94.33 & 94.29 & 95.63 \\
\cline { 2 - 7 } & Hard & 99.32 & 97.60 & 96.00 & 92.89 & 95.45 \\
\hline
\end{tabular}

Table 6 - Results of percentages of measured data found in the $95 \%$ confidence interval for acceleration for each trial and each runner. 


\begin{tabular}{|c|c|c|c|c|c|}
\hline & Male 1 & Male 2 & Male 3 & Female 1 & Female 2 \\
\hline Soft & 0.94 & 0.33 & 0.89 & 0.95 & 0.21 \\
\hline Medium & 0.86 & 0.33 & 0.84 & 0.82 & 0.96 \\
\hline Hard & 0.52 & 0.63 & 0.008 & 0.61 & 0.29 \\
\hline
\end{tabular}

Table 7-p-values of Durbin-Watson statistic to check for autocorrelation among residuals for each runner and for each acceleration trial intensity ("soft", "medium" and "hard")

\subsubsection{Testing for non autocorrelation of residuals}

In this subsection, we want to detect the presence of autocorrelation among residuals. In our case, the residuals is a vector defined by the difference between our measured data $a(t)$ and the estimated acceleration parameter from the Ornstein-Uhlenbeck model $(\hat{a})$. We perform the Durbin-Watson statistic on the following linear regression:

$$
a(t)-a\left(t-\Delta_{t}\right)=\hat{\theta}(a(t)-\hat{a}) \Delta_{t}+\eta(t)
$$

where $\eta(t)$ is the vector of residuals. If the model is correct, the residuals are independent and their distribution follows a normal distribution described as follows: $\mathcal{N}\left(0, \sigma^{2} \Delta t\right)$

In order to perform the Durbin-Watson statistic, we define the null hypothesis $\left(H_{0}\right)$ as follows: the residuals of the linear regression (4) are uncorrelated. The alternative hypothesis $\left(H_{1}\right)$ is that there is autocorrelation among the residuals. In order to test $H_{0}$ against $H_{1}$, we used the function dwtes ${ }^{1}$ from Matlab @. The results of the test are shown in Table 7 .

The range of $p-$ value is included between 0.08 and 0.98 . Given those results, we cannot reject the null hypothesis stating that the residuals are uncorrelated.

\subsubsection{Testing for stationarity}

In this subsection, we test for no unit root and wether the process of acceleration is stationary or not to insure that the estimated mean accelerations neither grow nor decrease over time. We define the null Hypothesis $\left(H_{0}\right)$ as follows: the unit root is present in the measured acceleration time series. The alternative hypothesis $\left(H_{1}\right)$ is defined as follows: the accelerations time series is trend-stationary. In order to test $H_{0}$ against $H_{1}$, we used the function adftest2 from Matlab@. The results of the test are shown in Table 8 Given those results, we reject the null hypothesis

\footnotetext{
${ }^{1}$ See the documentation on dwtest: https://fr.mathworks.com/help/stats/dwtest.html

${ }^{2}$ See the documentation on adftest: https://fr.mathworks.com/help/econ/adftest.html
} 


\begin{tabular}{|c|c|c|c|c|c|}
\hline & Male 1 & Male 2 & Male 3 & Female 1 & Female 2 \\
\hline Soft & $<1.0 \mathrm{e}-03$ & $<1.0 \mathrm{e}-03$ & $<1.0 \mathrm{e}-03$ & $<1.0 \mathrm{e}-03$ & $<1.0 \mathrm{e}-03$ \\
\hline Medium & $<1.0 \mathrm{e}-03$ & $<1.0 \mathrm{e}-03$ & $<1.0 \mathrm{e}-03$ & $<1.0 \mathrm{e}-03$ & $<1.0 \mathrm{e}-03$ \\
\hline Hard & $<1.0 \mathrm{e}-03$ & $<1.0 \mathrm{e}-03$ & $<1.0 \mathrm{e}-03$ & $<1.0 \mathrm{e}-03$ & $<1.0 \mathrm{e}-03$ \\
\hline
\end{tabular}

Table 8-p-value of Augmented Dickey-Fuller static to check for stationarity for each runner and for each acceleration trial intensity ("soft", "medium" and "hard")

stating that the simulation of acceleration is non-stationary.

\section{Discussion}

Our results showed that recreational runners are able to control their acceleration until exhaustion at three significantly different accelerations values perceived to be "soft", "medium" and "hard". Indeed, our data showed that runners are able to apply distinct, subjective acceleration values when so instructed and maintain constant acceleration until exhaustion, regardless of the acceleration value. In addition, our results showed that self-paced acceleration fitted the following Ornstein-Uhlenbeck process $d a_{t}=-\theta\left(a_{t}-a\right) d t+\sigma d W_{t}$ where $a$ is the mean acceleration, $\theta$ is the human ability for autocorrection, $\sigma$ the human variation and $a_{t}$ the measured acceleration. Indeed, a fine-grain analysis of the data shows that accelerations are obtained by the application of brief and frequent corrections designed to reach a mean target value a, and that there is no drift in the accelerations. Moreover, the fact that there is no unit-root in the acceleration time series shows that possible strong deviance from the mean value $a$ are rapidly corrected and vanishes by mean-reverting corrections. Further studies are now required for characterizing the deceleration of a recreational runner.

Given the fact that this is the first study having examine the human ability to maintain self-paced acceleration, we are going to discuss the two following points:

1. The self-paced acceleration range values compared with current use of acceleration protocols in laboratory.

2. The possible mechanisms allowing humans to control its body acceleration in accordance with prior results obtained on manual task. 


\subsection{The self-paced acceleration range values compared with current use of acceleration protocols in laboratory}

The range of these perceived acceleration values is in accordance with observed values in middledistance running [28]. Furthermore, these freely chosen accelerations also correspond to the imposed acceleration values frequently used in treadmill ramp protocols for determining VO2max 29, 30. In ramp protocols, the work rate is ramped up as a continuous increase and then a continuous acceleration. Given that the linearity of the oxygen uptake response is a major discriminating cardiovascular feature for assessing exercise intolerance, it is important to be sure that the work rate profile is linear and then the acceleration is constant. That is why the tests are currently performed on a treadmill. Even though manufacturers have developed a range of technologies for enabling ramp tests (e.g. controlled cycle ergometer), some subjects have difficulty walking and running on a treadmill and then reaching their maximum VO2 and speed at VO2max. It has been argued that the treadmill use induces a higher maximum metabolic rate and requires a mode of exercises that more closely approximates some activities of daily living [31, 32. In a much easier protocol, the present study shows that male and female middle-aged, recreational runners were able to self-monitor acceleration and thus reproduced an outdoor ramp protocol. Indeed, the present results showed that it is possible to apply a self-paced, ramp-like running protocol on a real running track. Regardless of the acceleration intensity level ("soft", "medium" or "hard"), the protocol uses a continuous change in speed and brings the subjects to exhaustion in approximately 2 minutes and 30 seconds to 20 minutes; this meets the criteria for clinical exercise testing issues by the relevant international organizations (American College of Sports Medicine 2000, American Thoracic Society 2003, [33, 34, 35]).

\subsection{The possible mechanisms allowing humans to control its body ac- celeration in accordance with prior results obtained on manual task}

Here we showed that human are accurately able to accelerate smoothly and constantly, with acceleration vs. time values close to $0 \mathrm{~m}^{-\mathrm{s}^{-2}}$ (even near to exhaustion). However, the question of how acceleration is controlled remains to be determined. There is still no studies having worked on the ability of self-pace its acceleration in locomotion. Therefore we can only discuss versus prior studies on arm and hand movement in catching tasks. In that tasks, neurophysiologists have demonstrated that the human brain imposes (in a top-down fashion) its rules of interpretation 
on sensory data. It transforms the perceived world according to rules of symmetry and stability and kinematic laws derived from principles of maximum smoothness [36]. They hypothesized that the first possibility was that humans can perceive distance very accurately [37]. It has been proved that humans possess efficient mechanisms for building an abstract representation of distances, which can be transferred from one perceptual context to another [37.

This paper seemed to show that whatever the variation in velocity, from "soft" acceleration intensity level at $0.0026 \mathrm{~m} . \mathrm{s}^{-2}$ to ten times that at $0.0253 \mathrm{~m} . \mathrm{s}^{-2}$ in the "hard" acceleration intensity level in self-paced acceleration trial, the acceleration was well controlled and steady, with a human ability to autocorrect and a minimal jerk value. The concept of "jerk-cost" has been validated for running and walking stride smoothness [38].

\section{Conclusion}

To the best of our knowledge, a human's ability to maintain constant acceleration in a conscious way until exhaustion has not yet been investigated. We therefore decided to test the hypothesis whereby humans are able to maintain a constant acceleration, regardless of the velocity and the magnitude of acceleration. Indeed, despite the large, published body of work on pacing strategy and speed control (especially concerning feedback vs. feed forward power output control and central vs. peripheral mechanisms), this is the first study to have examined acceleration control during running.

The main findings of this research are as follows:

1. The measured data seemed to show that runners are able to apply at least three distinct and subjective acceleration intensities when so instructed.

2. The measured data seemed to show that the runners tend towards reducing the time and distance to exhaustion between the "soft" and the "medium" instruction as well as between the "medium" and "soft" instructions.

3. The simulation using the Ornstein-Uhlenbeck process seemed to show that accelerations are obtained by the application of brief and frequent corrections designed to reach a mean target value $a$.

4. The simulation showed that the runners tend towards increasing the mean acceleration between the "soft" and the "medium" instruction as well as between the "medium" and "soft" instructions. 
Our results show that runners are able to control their acceleration until exhaustion at three significantly different accelerations values perceived to be "soft", "medium" and "hard". According to Newton's Second Law, humans could control their force of motion as it is directly proportional to the acceleration opening the door on future study related to human physiology and human energetic. We can think of two research opportunities go further. One would be to study the deceleration and see if humans are also able to control it. Once we successfully describe both acceleration and deceleration with mathematical models, we could think about alternating acceleration and deceleration phases to see how runners would react and study the sequence. The other would be gathering data from many runners, both males and females, in order to establish a predictive model for human acceleration. This could be done using Stochastic Differential Mixed-Effects Equations.

\section{Appendices}

Appendix A
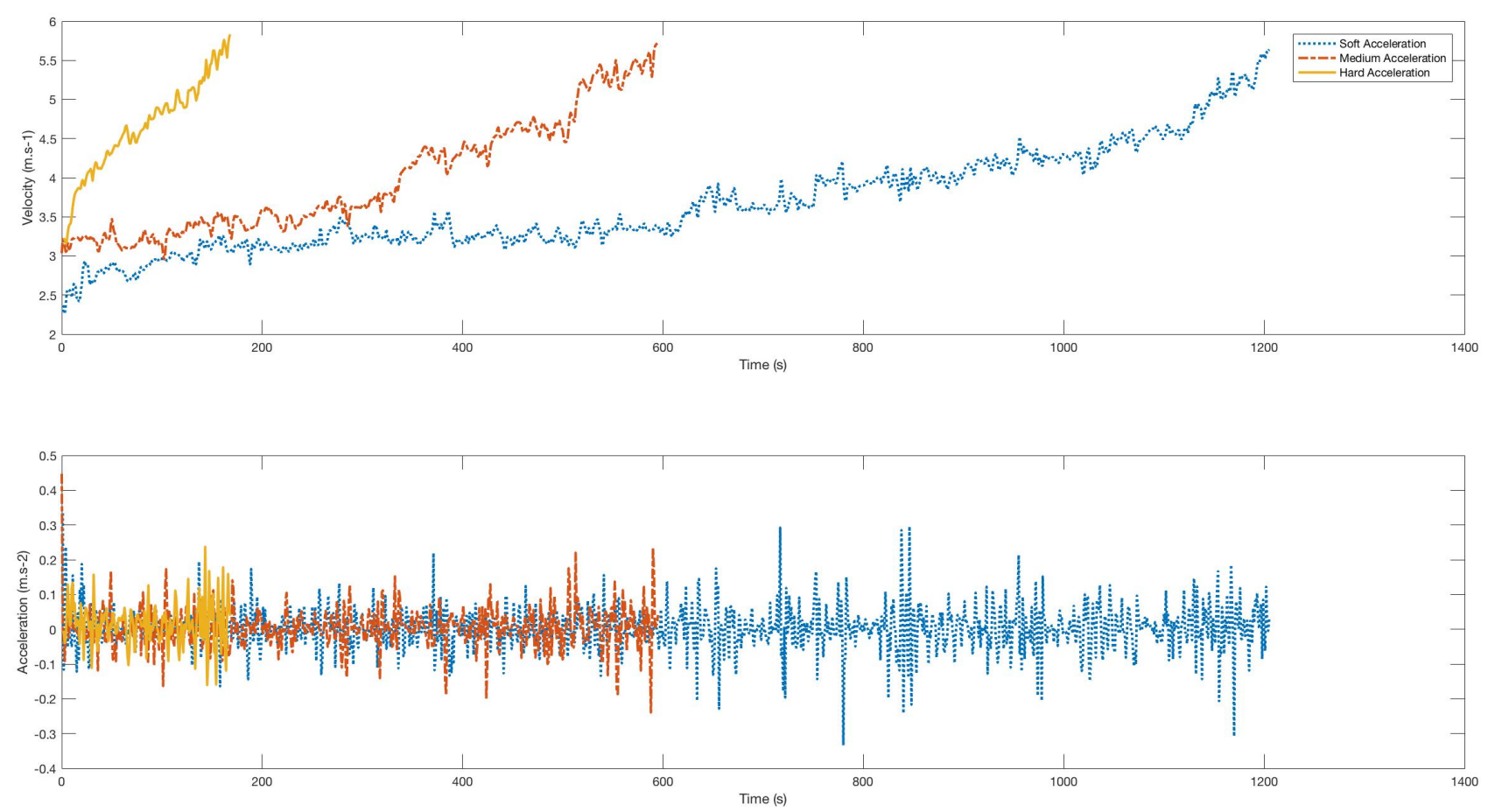
Figure 4 - Male Runner 2 - Top chart: Velocity (m/s) VS. Time (s); Bottom chart: Acceleration $\left(\mathrm{m} / \mathrm{s}^{2}\right)$ VS. Time (s) - Comparison of the three acceleration intensity instructions: "soft" (blue curve), "medium" (red curve) and "hard" (yellow curve)
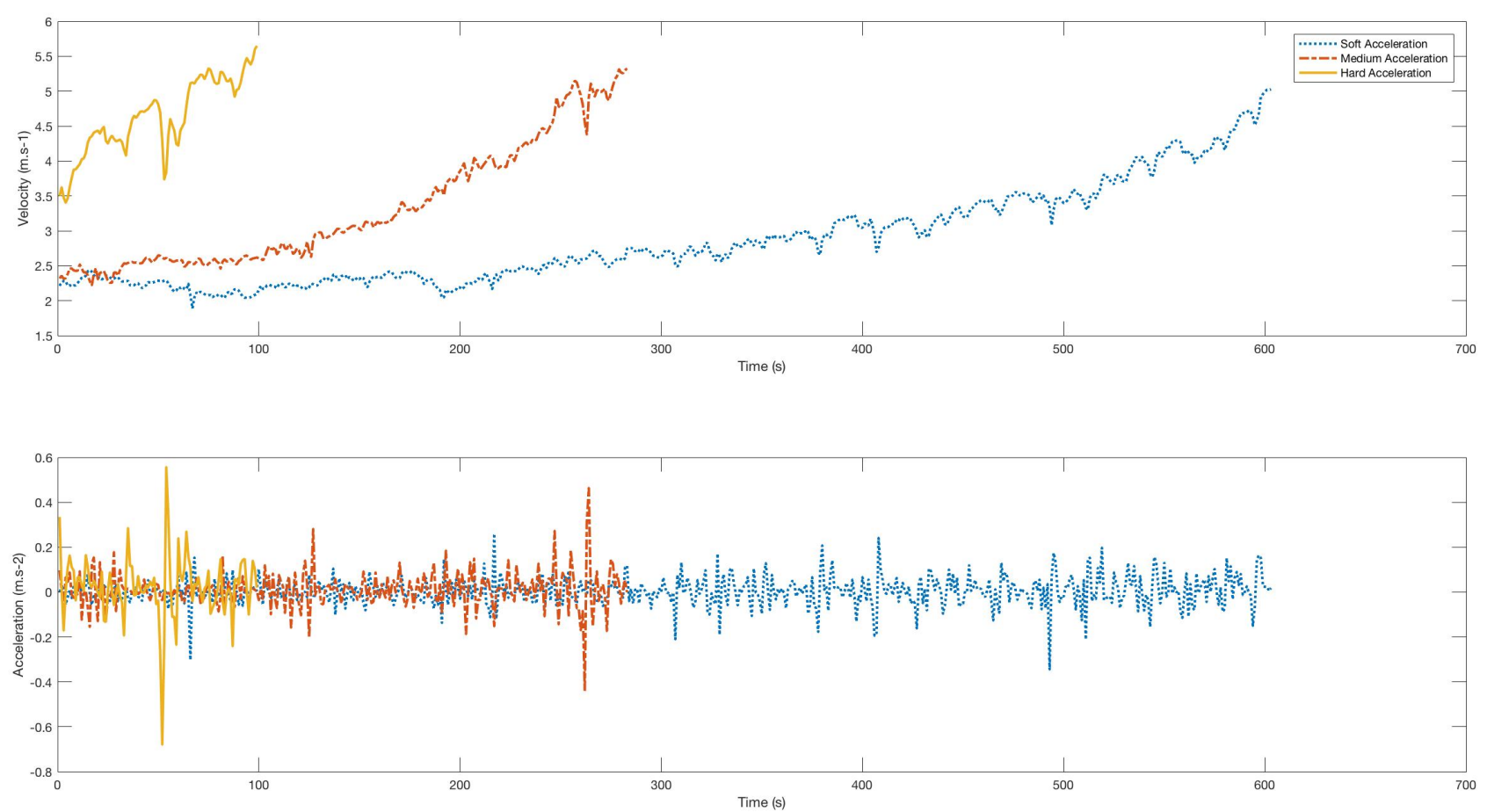

Figure 5 - Male Runner 3 - Top chart: Velocity (m/s) VS. Time (s); Bottom chart: Acceleration $\left(\mathrm{m} / \mathrm{s}^{2}\right)$ VS. Time (s) - Comparison of the three acceleration intensity instructions: "soft" (blue curve), "medium" (red curve) and "hard" (yellow curve) 

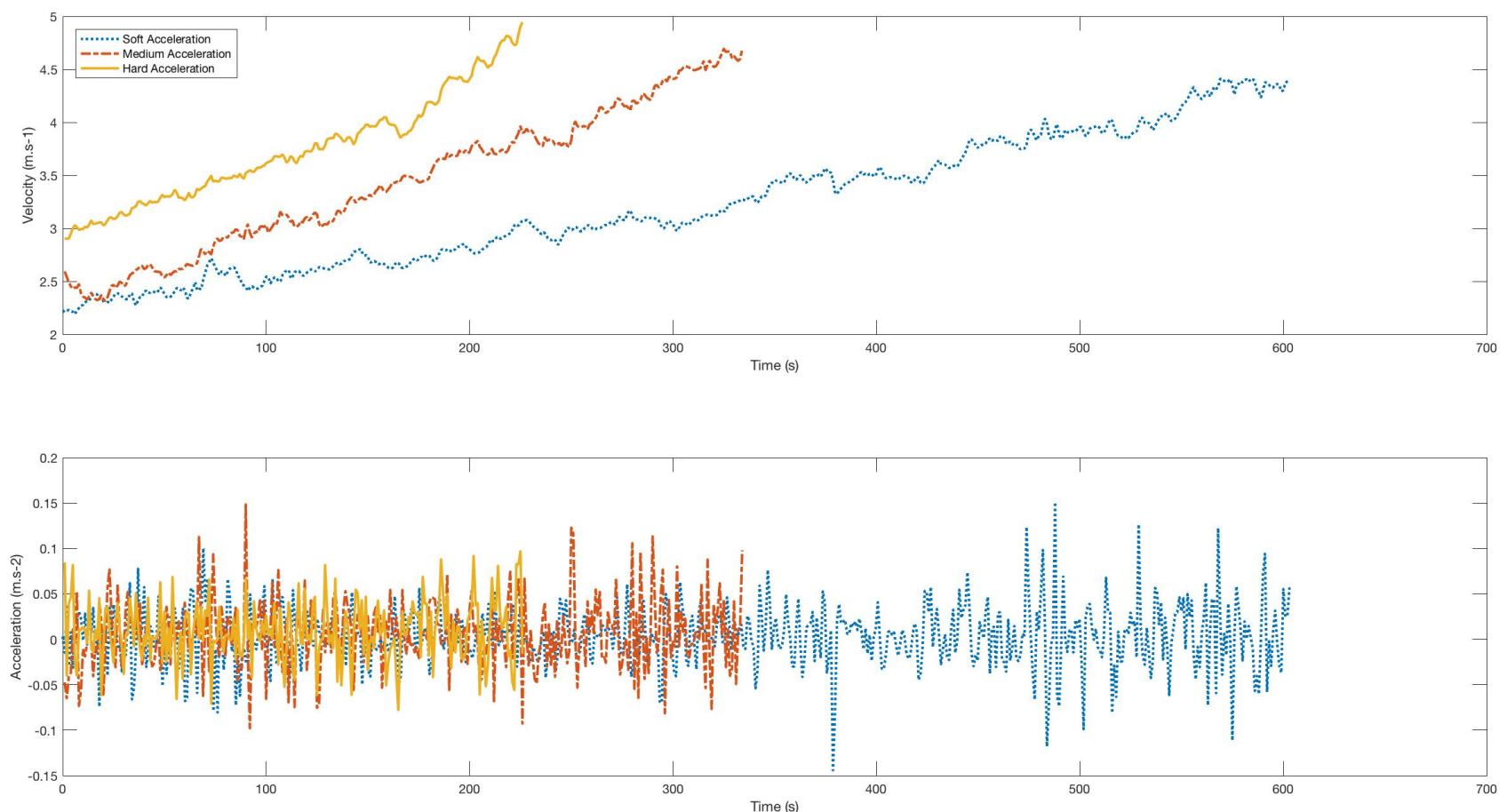

Figure 6 - Female Runner 1 - Top chart: Velocity (m/s) VS. Time (s); Bottom chart: Acceleration $\left(\mathrm{m} / \mathrm{s}^{2}\right)$ VS. Time (s) - Comparison of the three acceleration intensity instructions: "soft" (blue curve), "medium" (red curve) and "hard" (yellow curve) 

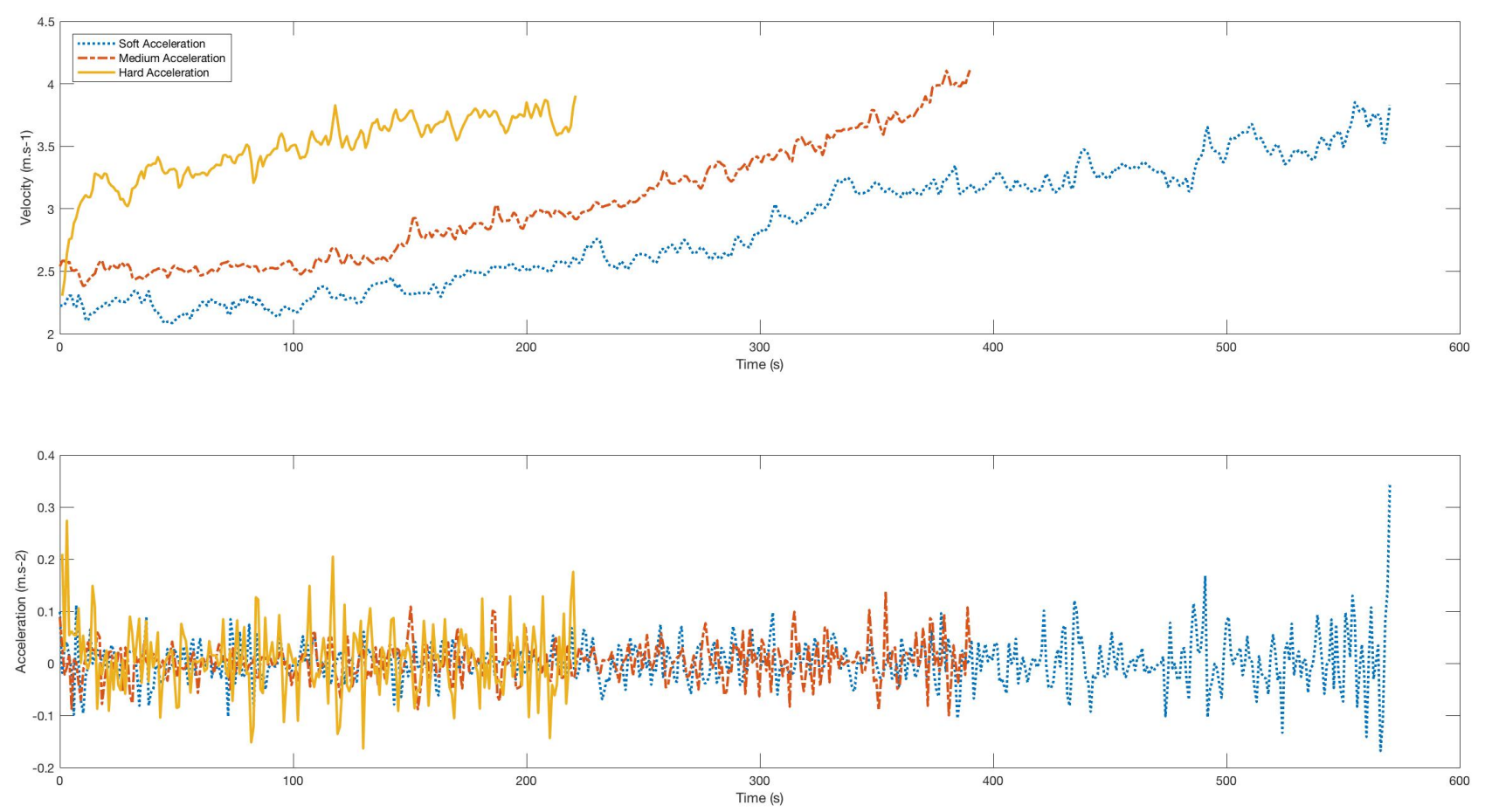

Figure 7-Female Runner 2 - Top chart: Velocity (m/s) VS. Time (s); Bottom chart: Acceleration $\left(\mathrm{m} / \mathrm{s}^{2}\right)$ VS. Time (s) - Comparison of the three acceleration intensity instructions: "soft" (blue curve), "medium" (red curve) and "hard" (yellow curve)

Appendix B 

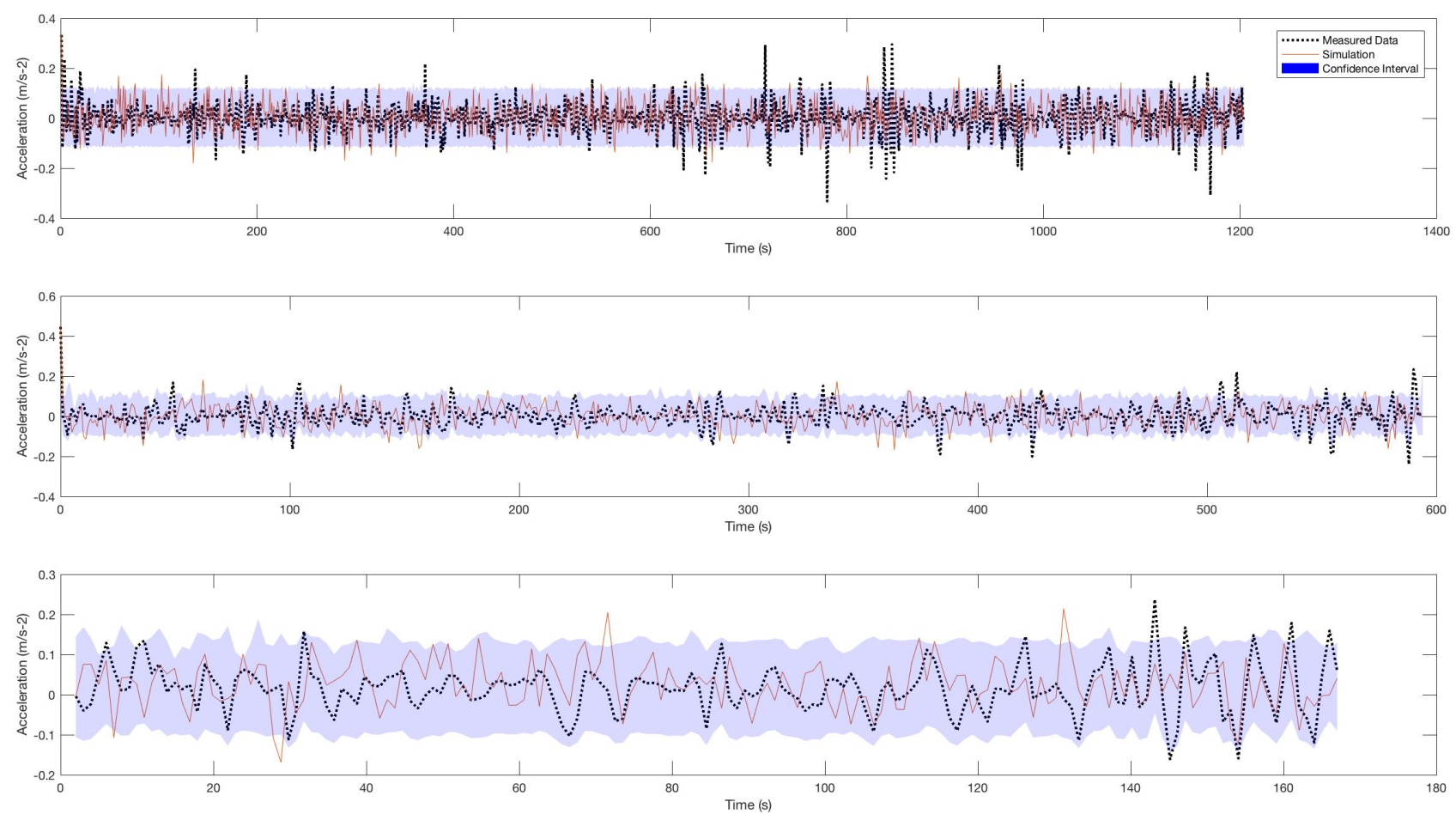

Figure 8 - Male Runner 2 - From top to bottom: Soft, Medium and Hard Acceleration Intensities Measured data VS. Ornstein-Uhlenbeck model 

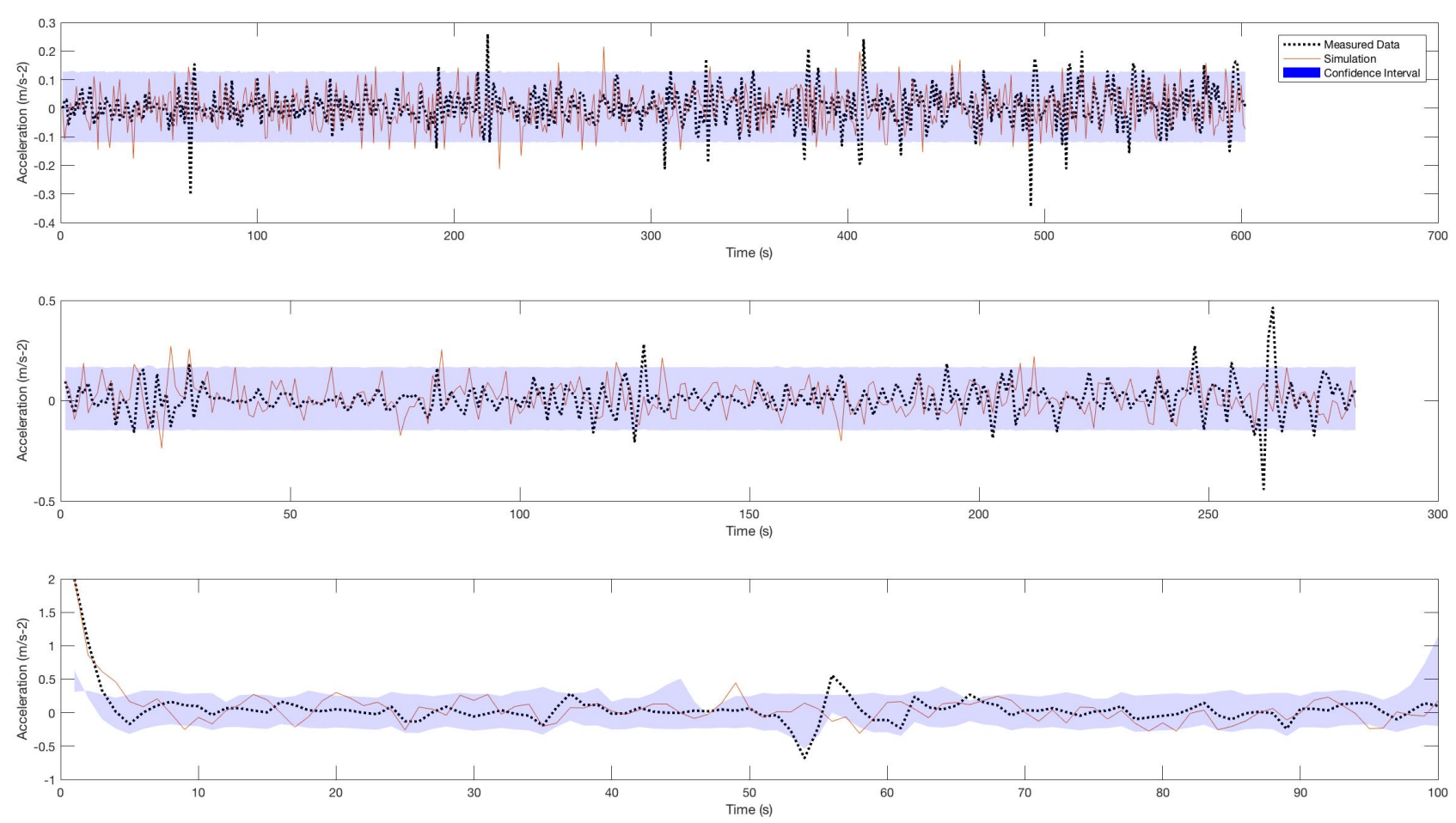

Figure 9 - Male Runner 3 - From top to bottom: Soft, Medium and Hard Acceleration Intensities Measured data VS. Ornstein-Uhlenbeck model 

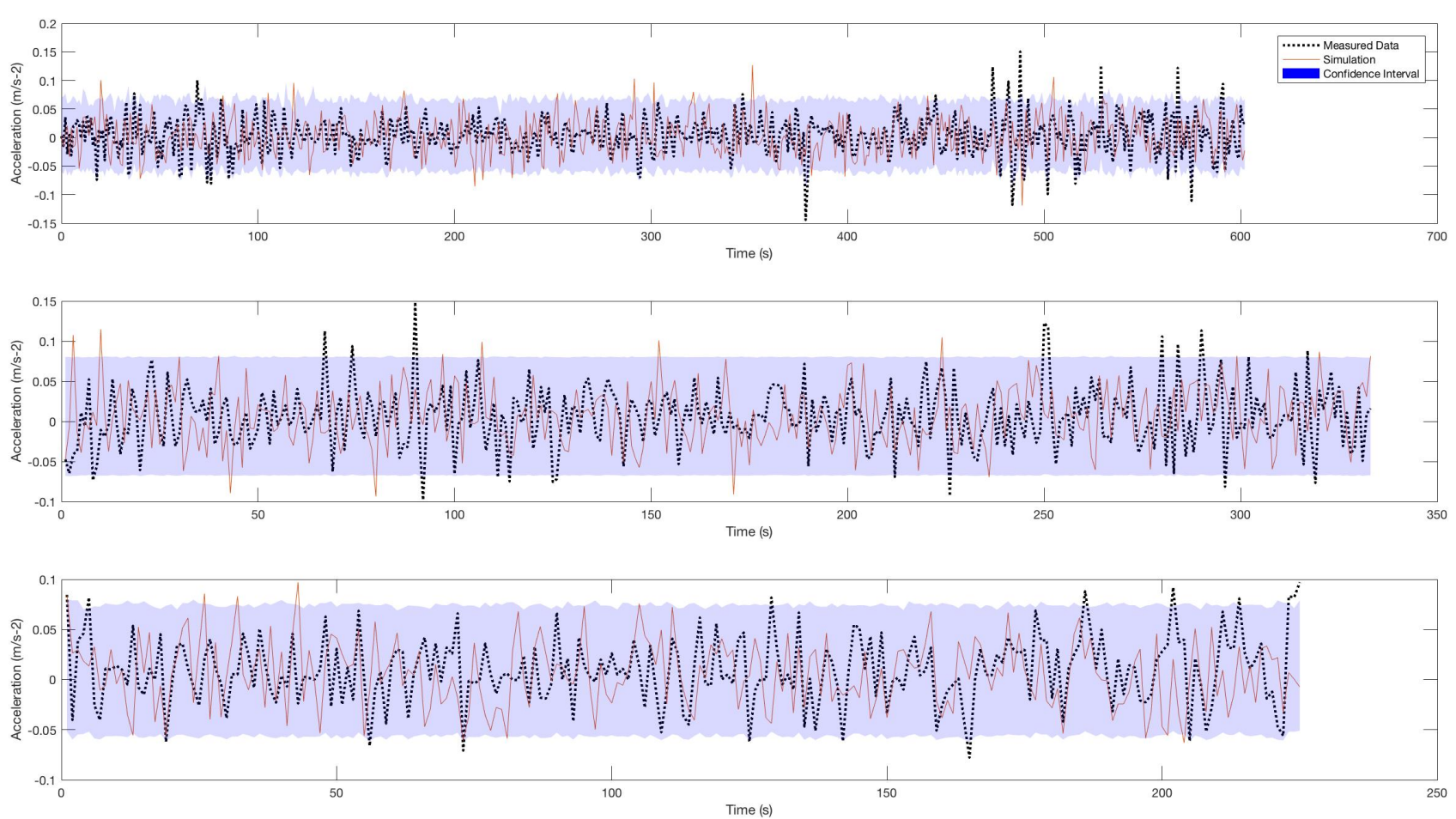

Figure 10 - Female Runner 1 - From top to bottom: Soft, Medium and Hard Acceleration Intensities Measured data VS. Ornstein-Uhlenbeck model 

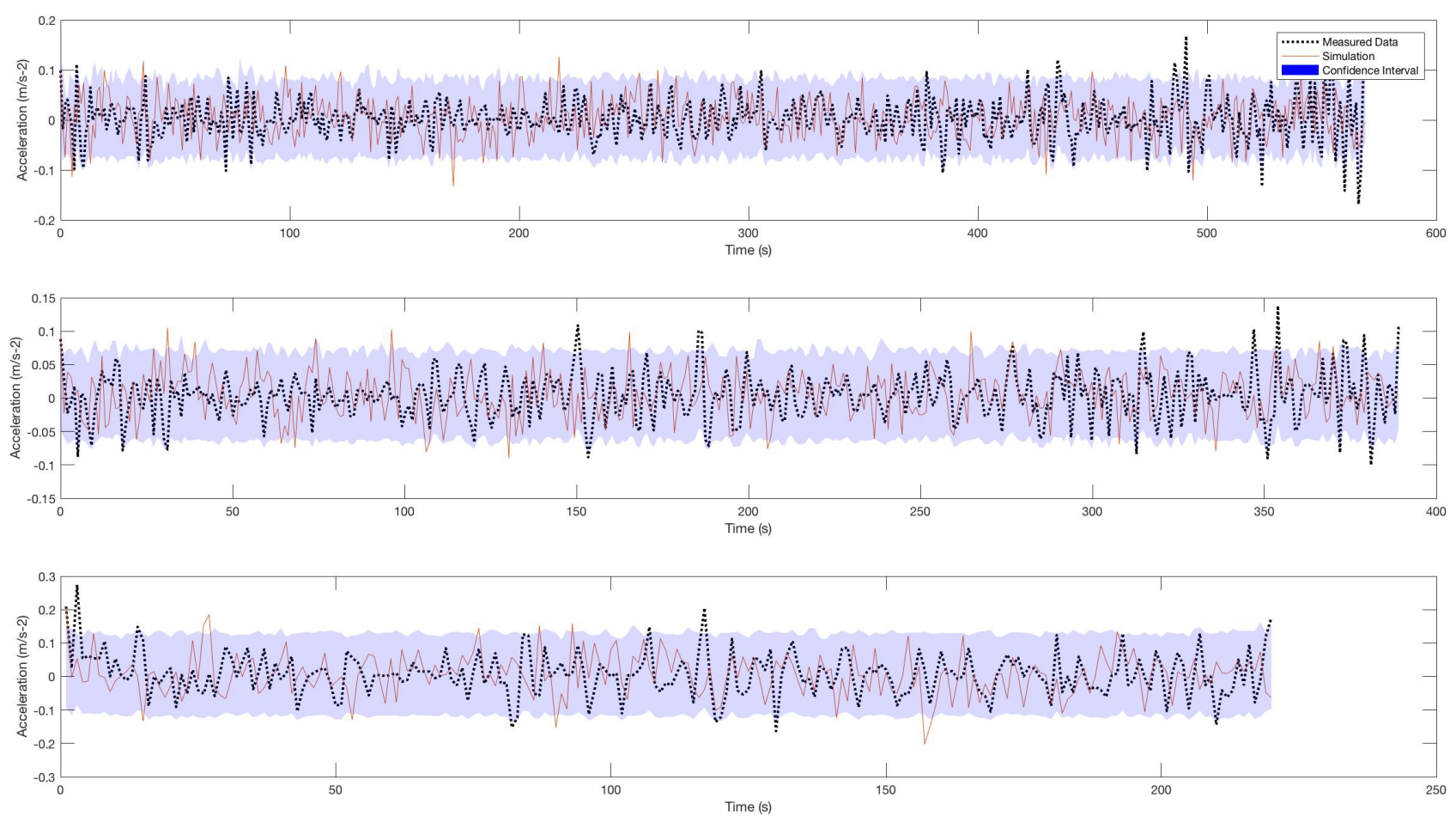

Figure 11 - Female Runner 2 - From top to bottom: Soft, Medium and Hard Acceleration Intensities Measured data VS. Ornstein-Uhlenbeck model

Appendix C 


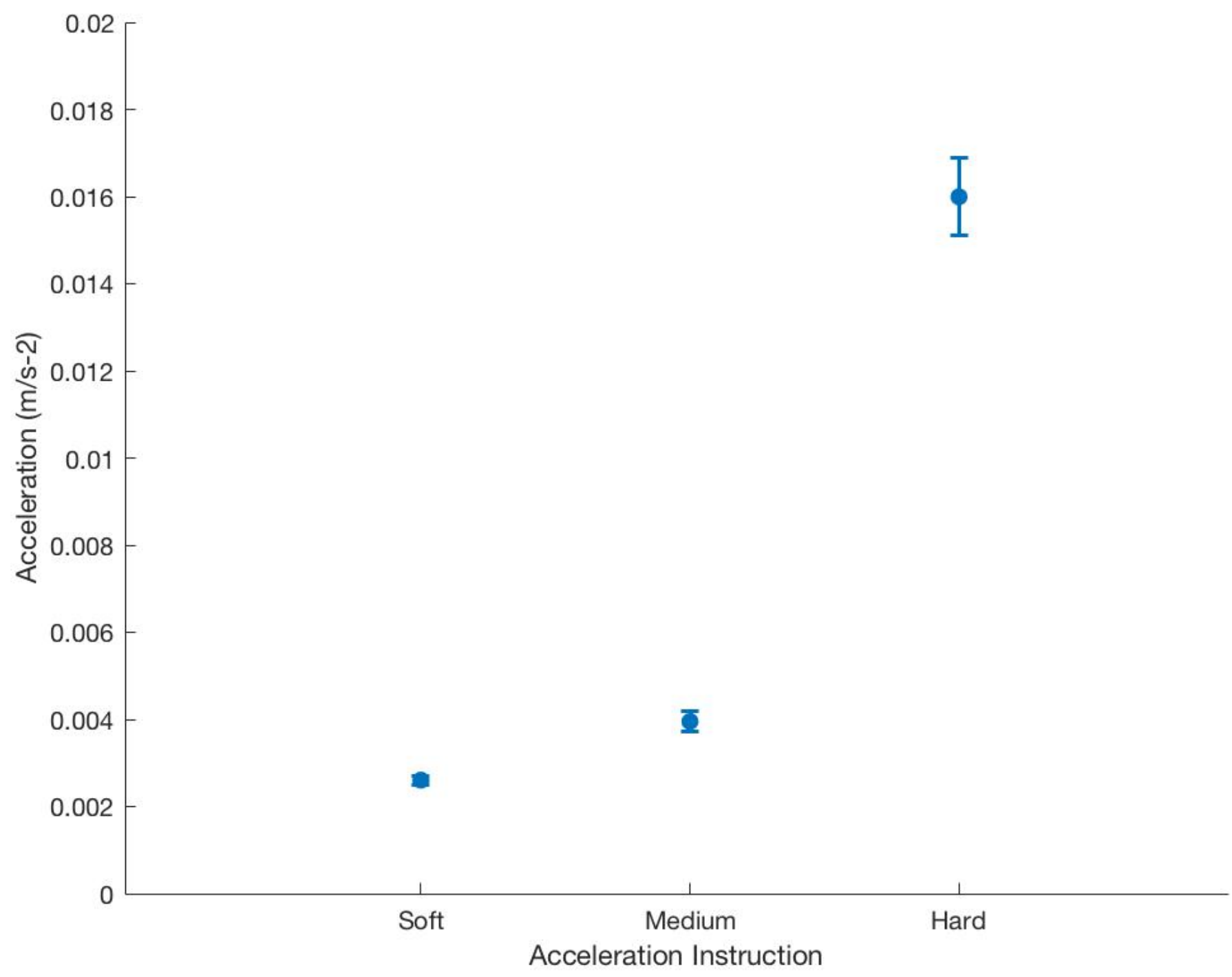

Figure 12 - Male Runner 2 - Comparison of the three estimated mean accelerations 


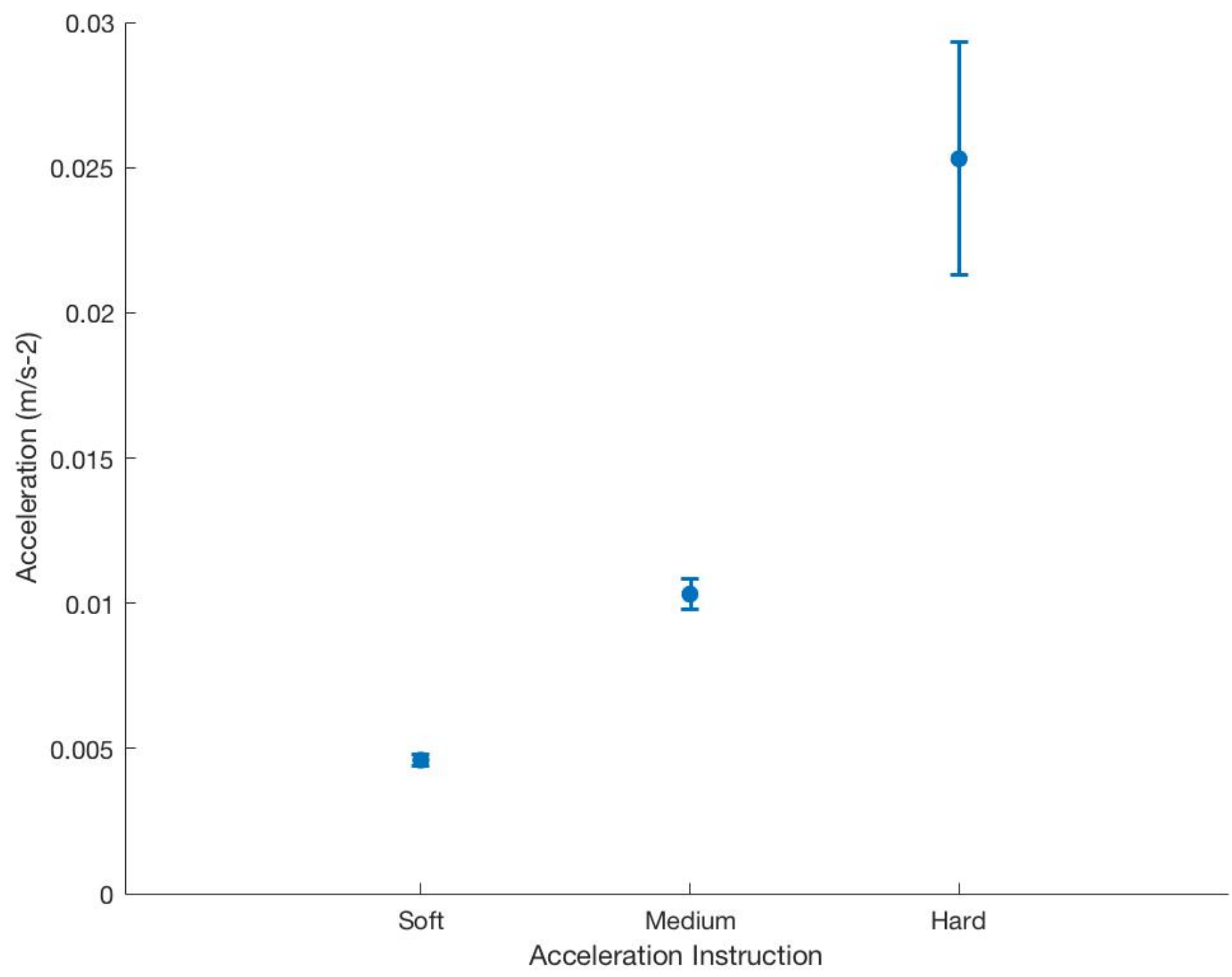

Figure 13 - Male Runner 3 - Comparison of the three estimated mean accelerations 


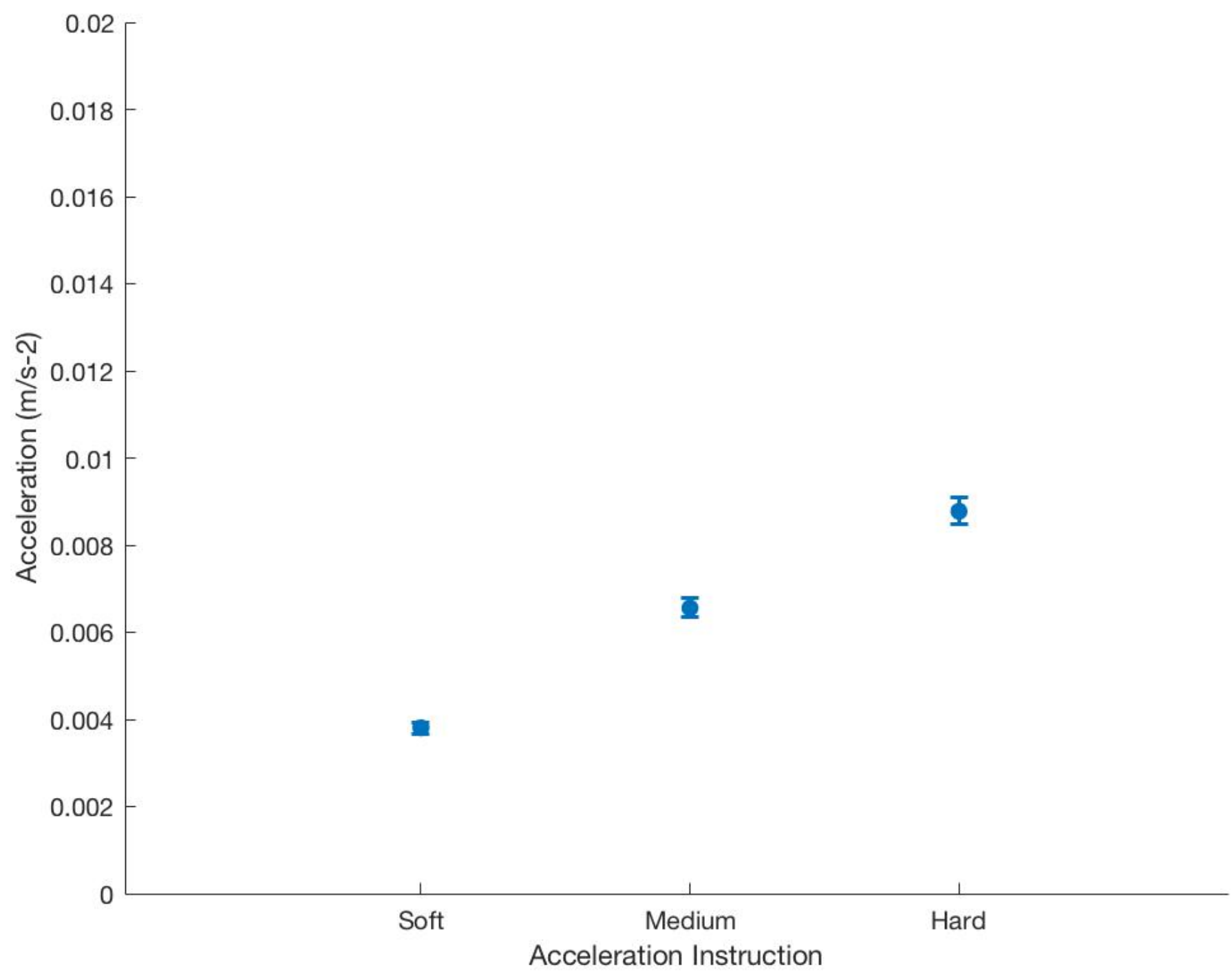

Figure 14 - Female Runner 1 - Comparison of the three estimated mean accelerations 


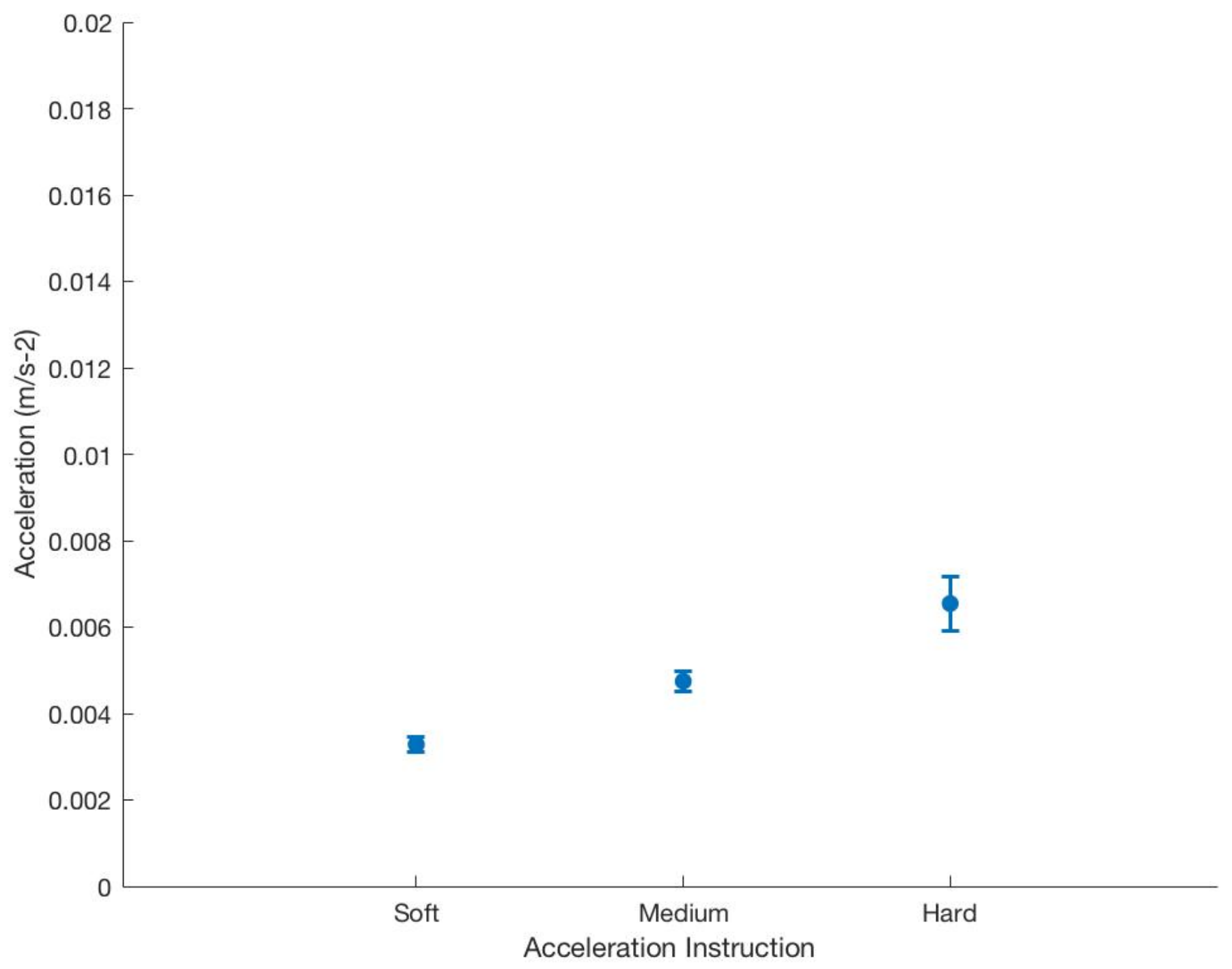

Figure 15 - Female Runner 2 - Comparison of the three estimated mean accelerations

\section{References}

[1] D. Bramble and D. Lieberman, "Endurance running and the evolution of homo," Nature, 2004.

[2] C. Foster, A. Snyder, N. Thompson, M. Green, M. Foley, and M. Schrager, "Effect of pacing 
strategy on cycle time trial performance," Medicine and Science in Sports and Exercise, 1993.

[3] C. Foster, M. Schrager, A. Snyder, and N. Thompson, "Pacing strategy and athletic performance," Sports Medicine, 1994.

[4] J. de Koning, C. Foster, A. Bakkum, S. Kloppenburg, C. Thiel, T. Joseph, J. Cohen, and J. Porcari, "Regulation of pacing strategy during athletic competition," PLos One, vol. 6, pp. 1-6, 012011.

[5] V. Billat, J. Slawinski, M. Danel, and J. Koralsztein, "Effect of free versus constant pace on performance and oxygen kinetics in running," Medicine and Science in Sports and Exercise, 2001.

[6] D. Bishop, D. Bonetti, and B. Dawson, "The influence of pacing strategy on vo2 and supramaximal kayak performance," Medicine and Science in Sports and Exercise, 2002.

[7] L. Sandals, D. Wood, S. Draper, and D. James, "Influence of pacing strategy on oxygen uptake during treadmill middle-distance running," Sports Medicine, 2006.

[8] R. Tucker, M. Lambert, and T. Noakes, "An analysis of pacing strategies during men's world-record performances in track athletics," International Journal of Sports Physiology and Performance, 2006.

[9] R. Tucker and T. Noakes, "The physiological regulation of pacing strategy during exercise: a critical review," British Journal of Sports Medicine, 2009.

[10] J. Hunter, R. Marshall, and P. McNair, "Relationships between ground reaction force impulse and kinematics of sprint-running acceleration," Journal of Applied Biomechanics, 2005.

[11] T. Flash and N. Hogan, "The coordination of arm movements: an experimentally confirmed mathematical model," Journal of Neuroscience, 1985.

[12] R. Tucker, A. Bester, E. Lambert, T. Noakes, C. Vaughan, and A. S. C. Gibson, "Non random fluctuations in power output during self paced exercise," British Journal of Sports Medicine, 2006.

[13] T. Noakes, A. S. C. Gibson, and E. Lambert, "From catastrophe to complexity: a novel model of integrative central neural regulation of effort and fatigue during exercise in humans," British Journal of Sports Medicine, 2004. 
[14] C. Abbiss and P. Laursen, "Describing and understanding pacing strategies during athletic competition," Sports Medicine, 2008.

[15] J. de Koning, C. Foster, A. Bakkum, S. Kloppenburg, C. Thiel, T. Joseph, J. Cohen, and J. Porcari, "Regulation of pacing strategy during athletic competition," Plos ONE, 2011.

[16] Ansley, Les, Noakes, Timothy, Robson-Ansley, Paula, S. C. Gibson, and Alan, "Anticipatory pacing strategies during supra-maximal exercise lasting more than $30 \mathrm{~s}$," Medicine and Science in Sports and Exercise, 2004.

[17] V. Billat, L. Mille-Hamard, Y. Meyer, and E. Wesfreid, "Detection of changes in the fractal scaling of heart rate and speed in a marathon race," Physica A, 2009.

[18] V. Billat, J. Slawinski, M. Danel, and J. Koralsztein, "Effect of free versus constant pace on performance and oxygen kinetics in running," Medicine and Science in Sports and Exercise, 2001.

[19] M. Ely, D. Martin, S. Cheuvront, and S. Montain, "Effect of ambient temperature on marathon pacing is dependent on runner ability," Medicine and Science in Sports and Exercise, 2008.

[20] T. Haney and J. Mercer, "A description of variability of pacing in marathon distance running," International Journal of Exercise Science, 2011.

[21] G. Uhlenbeck and L. Ornstein, "On the theory of the brownian motion," Physical review, 1930.

[22] R. Schobel and J. Zhu, "Stochastic volatility with an ornstein-uhlenbeck process: An extension," Review of Finance, 1999.

[23] L. Ricciardi and L. Sacerdote, "The ornstein-uhlenbeck process as a model for neuronal activity," Biological Cybernetics, 1979.

[24] J. C. Barbero-Alvarez, A. Coutts, J. Granda, V. Barbero-Alvarez, and C. Castagna, "The validity and reliability of a global positioning satellite system device to assess speed and repeated sprint ability (rsa) in athletes," Journal of Science and Medicine in Sport, 2009.

[25] P. Larsson and K. Henriksson-Larsen, "The use of dgps and simultaneous metabolic measurements during orienteering," Medicine and Science in Sports and Exercise, 2001. 
[26] V. Billat, P. Lepretre, A. Heugas, and J. Koralsztein, "Energetics of middle-distance running performances in male and female junior using track measurements.," Japanese Journal of Physiology, 2004.

[27] D. Gillespie, "Exact numerical simulation of the ornstein-uhlenbeck process and its integral," Physical Review E, 1996.

[28] V. Billat, L. Hamard, J. Koralsztein, and R. Morton, "Differential modeling of anaerobic and aerobic metabolism in the 800-m and 1,500-m run," Journal of Applied Physiology, 2009.

[29] J. Myers and D. Bellin, "Ramp exercise protocols for clinical and cardiopulmonary exercise testing," Sports Medicine, 2000.

[30] J. Porszasz, R. Casaburi, A. Somfay, L. Woodhouse, and B. Whipp, "A treadmill ramp protocol using simultaneous changes in speed and grade," Medicine and Science in Sports and Exercise, 2003.

[31] B. Balke and R. Ware, "An experimental study of physical fitness of air force personnel," United States Armed Forces Medical Journal, 1959.

[32] V. Froelicher and J. Myers, Exercise and the Heart. Elsevier, 2000.

[33] J. Myers, N. Buchanan, D. Smith, J. Neutel, E. Bowes, D. Walsh, and V. Froelicher, "Individualized ramp treadmill. observations on a new protocol," Chest, 1992.

[34] R. Gibbons, G. Balady, J. Beasley, J. Bricker, W. Duvernoy, V. Froelicher, D. Mark, T. Marwick, B. McCallister, P. J. Thompson, W. Winters, F. Yanowitz, J. Ritchie, R. Gibbons, M. Cheitlin, K. Eagle, T. Gardner, A. J. Garson, R. Lewis, R. O'Rourke, and T. Ryan, "Guidelines for exercise testing: a report of the american college of cardiology/ american heart association task force on practice guidelines," Journal of the American College of Cardiology, 1997.

[35] P. Will and J. Walter, "Exercise testing: improving performance with a ramped bruce protocol," American Heart Journal, 1999.

[36] A. Berthoz, Neurobiology of "Umwelt". Springer, 2009.

[37] M. Mossio, M. Vidal, and A. Berthoz, "Traveled distances: new insights into the role of optic flow," Vision Research, 2008. 
[38] A. Hreljac, "Stride smoothness evaluation of runners and other athletes," Gait Posture, 2000. 